\title{
Antimicrobial and antibiofilm activities of Cichorium intybus: a review
}

\author{
A tividade antimicrobiana e antibiofilme da Cichorium intybus: uma revisão \\ Actividad antimicrobiana y antibiopelícula de Cichorium intybus: una revisión
}

Received: 01/05/2022 | Reviewed: 01/11/2022 | Accept: 01/18/2022 | Published: 01/19/2022

\author{
Mariana Sobreira Bezerra \\ ORCID: https://orcid.org/0000-0003-2868-2974 \\ Federal University of Santa Maria, Brazil \\ E-mail: marisbezerra1@gmail.com \\ Kamila Said Zeferino \\ ORCID: https://orcid.org/0000-0002-1364-0782 \\ Federal University of Ceará, Brazil \\ E-mail: kamilasaid@hotmail.com \\ Larissa Dutra Menezes \\ ORCID: https://orcid.org/0000-0003-1843-247X \\ Federal University of Ceará, Brazil \\ E-mail: larissadutra@alu.ufc.br \\ Aline Sobreira Bezerra \\ ORCID: https://orcid.org/0000-0003-1370-9564 \\ Federal University of Santa Maria, Brazil \\ E-mail: alinecelo@hotmail.com \\ Leonardo Quintana Soares Lopes \\ ORCID: https://orcid.org/0000-0003-3793-5216 \\ Federal University of Santa Maria, Brazil \\ E-mail: leonardoquintanalopes@gmail.com \\ Flávia Kolling Marquezan \\ ORCID: https://orcid.org/0000-0003-1505-2447 \\ Franciscana University, Brazil \\ E-mail: flaviamarquezan @ gmail.com \\ Patricia Kolling Marquezan \\ ORCID: https://orcid.org/0000-0001-5061-6039 \\ Federal University of Santa Maria, Brazil \\ E-mail: patimarquezan@ hotmail.com
}

\begin{abstract}
The plant Cichorium intybus L., also known as Chicory, is found in different parts of the world and has a rich phytochemical composition. Within its spectrum of action, the plant has been studied about its performance against pathogenic microorganisms and biofilm. Therefore, the objective of the present study is to realize a literature review on the antimicrobial and antibiofilm actions of the species Cichorium intybus. For this, searches were carried out in the databases PubMed / MEDLINE, EMBASE, Gale - Academic OneFile and Science Direct for articles published until August 2020, using the MeSH descriptors "Anti-infective agents", "Dentistry" and "Periodontal disease" and its derivatives together with the keyword "Cichorium intybus" and its variants. A total of 998 results were found. After a critical reading of titles and abstracts, 18 articles were selected for the preparation of the study. The review includes articles published without time restriction and that approach the species Cichorium intybus with antimicrobial and antibiofilm action. Case reports and literature reviews were excluded. Chicory has a rich variety of chemical compounds that ensure it a wide range of uses, presenting action against bacteria, fungi and viruses, especially the Hepatitis B virus. Therefore, Almeirão has a good antimicrobial and antibiofilm performance in vitro, however, more studies are needed to standardize the study methodology, in addition to elucidating the clinical practice of the plant.
\end{abstract}

Keywords: Anti-infective agents; Antibiofilm; Antimicrobial; Cichorium intybus.

\section{Resumo}

A planta Cichorium intybus L., também conhecida como Chicória, é encontrada em diferentes partes do mundo e possui uma rica composição fitoquímica. Dentro de seu espectro de ação, a planta vem sendo estudada quanto ao seu desempenho contra microrganismos patogênicos e biofilme. Portanto, o objetivo do presente estudo é realizar uma revisão da literatura sobre as ações antimicrobiana e antibiofilme da espécie Cichorium intybus. Para isso, foram realizadas buscas nas bases de dados PubMed / MEDLINE, EMBASE, Gale - Academic OneFile e Science Direct para artigos publicados até agosto de 2020, utilizando os descritores MeSH "Anti-infective agentes", "Dentistry" and "Periodontal disease" e seus derivados juntamente com a palavra-chave "Cichorium intybus" e suas variantes. Um total de 998 resultados foram encontrados. Após a leitura crítica dos títulos e resumos, 18 artigos foram selecionados para a elaboração do estudo. A revisão inclui artigos publicados sem restrição de tempo e que abordam a espécie 
Cichorium intybus com ação antimicrobiana e antibiofilme. Relatos de caso e revisões de literatura foram excluídos. A chicória possui uma rica variedade de compostos químicos que lhe garantem uma ampla gama de utilizações, apresentando ação contra bactérias, fungos e vírus, principalmente o vírus da Hepatite B. Portanto, o Almeirão apresenta um bom desempenho antimicrobiano e antibiofilme in vitro, porém, mais estudos são necessários para padronizar a metodologia do estudo, além de elucidar a prática clínica da planta.

Palavras-chave: Agentes anti-infecciosos; Antibiofilme; Antimicrobianos; Cichorium intybus.

\section{Resumen}

La planta Cichorium intybus L., también conocida como a chicoria, se encuentra en diferentes partes del mundo y tiene una rica composición fitoquímica. Dentro de su espectro de acción, se ha estudiado la planta en cuanto a su comportamiento frente a microorganismos patógenos y biofilm. Por tanto, el objetivo de este estudio es realizar una revisión de la literatura sobre las acciones antimicrobianas y antibiofilm de la especie Cichorium intybus. Para ello, se realizaron búsquedas en las bases de datos PubMed / MEDLINE, EMBASE, Gale - Academic OneFile y Science Direct de artículos publicados hasta agosto de 2020, utilizando los descriptores MeSH "Agentes antiinfecciosos", "Odontología" y "Enfermedad periodontal" y sus derivados junto con la palabra clave "Cichorium intybus" y sus variantes. Se encontraron un total de 998 resultados. Luego de la lectura crítica de los títulos y resúmenes, se seleccionaron 18 artículos para la elaboración del estudio. La revisión incluye artículos publicados sin restricción de tiempo y que abordan la especie Cichorium intybus con acción antimicrobiana y antibiofilm. Se excluyeron los informes de casos y las revisiones de la literatura. La achicoria tiene una rica variedad de compuestos químicos que garantizan una amplia gama de usos, actuando contra bacterias, hongos y virus, especialmente el virus de la Hepatitis B. Por lo tanto, Almeirão tiene un buen desempeño antimicrobiano y antibiofilm in vitro, sin embargo, se necesitan más estudios para estandarizar la metodología de estudio, además de dilucidar la práctica clínica de la planta.

Palabras clave: Agentes anti-infecciosos; Antibiopelícula; Antimicrobianos; Cichorium intybus.

\section{Introduction}

In the last years, the search for natural alternatives to conventional treatments has grown vertiginously. Since antiquity, records of the use of compounds from natural agents, such as plants, were found for different medicinal purposes (Eldin \& Dunford, 2011). The phenomenon of phytotherapy has invited an increasing number of people and studies, because it has natural compounds that have, among other advantages, greater bioavailability, greater accessibility for acquisition by the population, less toxic effects due to biocompatibility and, finally, pharmacological results equivalent or better than conventional ones (Francisco, 2010).

Among the studies in the field of phytotherapy, there is the species Cichorium intybus L., popularly known for “Chicory” (Shaikh et al., 2016; Khatami et al., 2018) or "Purple Swallow” (Mazzaglia et al., 2020) or “Almeirão" (Bezerra et al., 2017). The genus Cichorium, to which the species belongs, is found in various places in the world, is more common in the region of Europe and Asia. Its use is reported in Greece, China and India due to its broad medicinal spectrum (Brieudes et al., 2016; Aisa et al., 2020). Of these uses in traditional medicine, the use of plant roots in the treatment of wounds, stings of venomous animals, such as snakes and scorpions, skin diseases, in addition to anti-inflammatory, antiangiogenic and antinociceptive, antiprotozoal actions, potentially anticancer and, according to studies in rats, has a protective effect against the immunotoxicity of alcohol (Kim et al., 2002; Lee et al., 2015; Kandil et al, 2019; Woolsey et al., 2019; Aisa et al., 2020).

Its composition rich in phenylpropenoids, flavonoids, polysaccharides (such as inulin), sesquiterpenoids, triterpenoids, steroids, proteins, lipids, nitrogenous components (such as caffeine) and organic acids may be the reason for the variety of actions to which the plant is attributed (Daglia et al., 2011; Bezerra et al., 2017; Aisa et al., 2020). Among the organic acids of the species, chicoric acid demonstrated antiviral action against the Hepatitis B virus and Human Immunodeficiency Virus (HIV). Caftáric acid is found in higher concentrations than in other plant species researched in the literature (Brieudes et al., 2016).

In addition, evidence of the use of the species within the dental field is also found, such as in the increase of salivary secretion in cases of xerostomia (Chamami et al., 2011), in the fight against caries disease by inhibiting the adhesion of the bacterial species Streptococcus mutans (Gazzani et al., 2012; Signoretto et al., 2013), in the control of the species 
Pseudomonas aeruginosa, related to patients affected with nosocomial pneumonia (Petrovic et al., 2004; Souto et al., 2014), adjuvant to the treatment of periodontal disease, aiming to control this condition (Babaei et al., 2018) and in the antibacterial activity against Prevotella intermedia and Actinomyces naeslundii, species related to gingivitis (Canesi et al., 2012).

Given the above, the objective of the present study was to evaluate the antimicrobial and antibiofilm action of bitter chicory from the existing literature, focusing on activities of interest in dentistry.

\section{Methodology}

The literature review followed the precepts of the exploratory study, through a bibliographic search in books and scientific articles on the subject. The qualitative method was used by the researchers because it allows the interpretation and issue of their opinions about what is studied (Pereira et al., 2018). Different searches were carried out, including articles that addressed the plant's antibiofilm or antimicrobial properties, without time restrictions but the articles should be published until 2020, august. The searches were carried out by a single researcher in the period of September 2020.

Literature reviews and books relevant to the topic were used in the body of the text, contributing to the discussion of the results. Articles were excluded by the exclusion criteria (escape from the theme, duplicates, not available in full) until reaching the articles included in the literature review. Electronic searches were performed in the PubMed / MEDLINE, EMBASE, Gale - Academic OneFile and Science Direct databases for articles published until August 2020, in addition to the complementary use of Google Scholar.

In the PUBMED database, the first research was carried out by combining the MeSH descriptors "Anti-infective agents" "Dentistry" and "Periodontal disease" and its derivatives, together with the keyword "Cichorium intybus" and synonyms joined with Boolean operator "AND". In total, 315 articles were found for further analysis of the title and abstract.

In the EMBASE library with terms cataloged in Emtree, 309 studies were found. Finally, the latest searches were carried out in the Science Direct and Gale - Academic OneFile databases using a combination of keywords resulting in 179 and 193 articles, respectively.

The flowchart (Figure 1) presented describes the number of articles found, how many excluded by the exclusion criteria (escape from the theme, duplicates, not available in full) until reaching the articles included in the literature review. Selection according to criteria was fundamental for the best reliability and power of generalization of the conclusions of this study. 


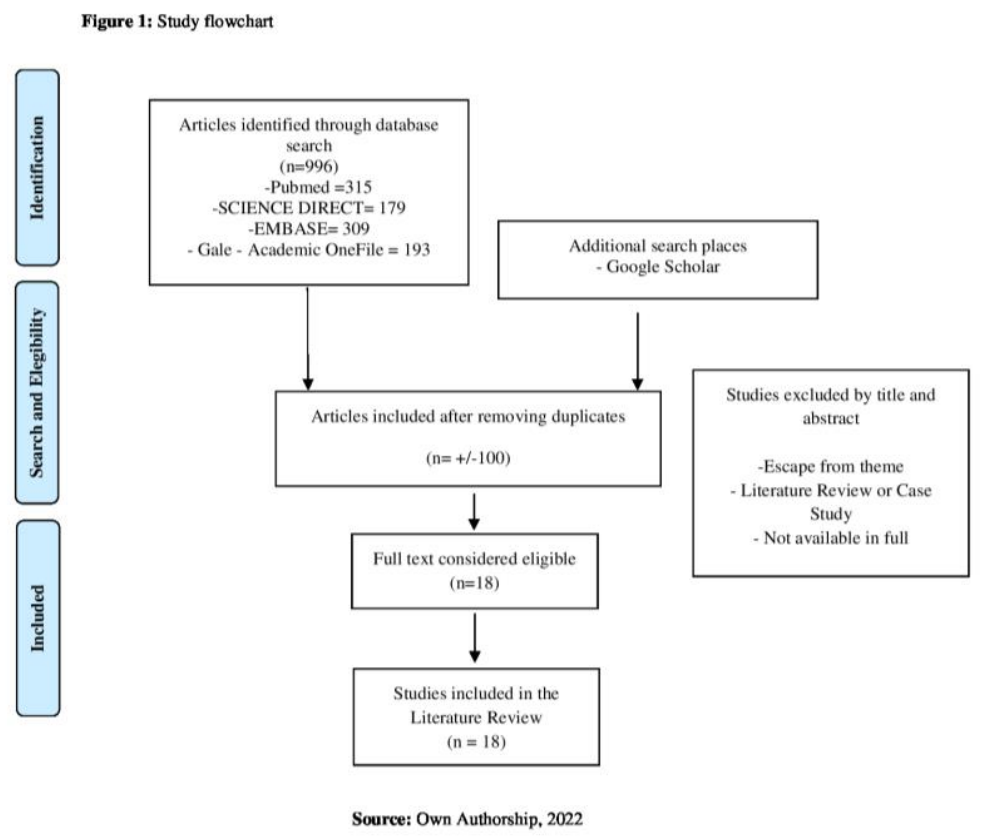

\section{Results and Discussion}

After the vast literature search, the articles were selected by title and abstract, subsequently eliminating duplicates and performing a critical reading of the studies considered eligible (998 articles). Articles were excluded by the exclusion criteria (escape from the theme, duplicates, not available in full) until reaching the articles included in the literature review. From the total of studies found, 18 scientific articles were selected that contemplated the plant's antimicrobial and antibiofilm action for discussion of experimental studies, in addition to ten articles (five in vitro studies and five literature reviews) and two books to complement the results. The selection following criteria was fundamental for the better reliability and generalizability of the conclusions of this study.

\section{Phytochemical characteristics of the species Cichorium intybus $L$.}

The plant Cichorium intybus L. belongs to the family Asteraceae. It is a small biennial or perennial fragrant herb, which is very common in regions in Europe, North America, Australia and Iraq. Elsewhere, such as in South Africa and Chile, this species is widely used to produce coffee substitutes from the dried, roasted and ground roots of Chicory, which results in a healthier and more affordable drink as a final result. Its leaves are also used in cooking, being consumed as cooked or raw vegetables. In general, fresh chicory contains inulin $(68 \%)$, sucrose $(14 \%)$, protein $(6 \%)$, cellulose $(5 \%)$, ash $(4 \%)$ and other compounds (3\%) in contrast to dry chicory, which contains inulin (98\%) and other compounds (2\%) (Nwafor et al., 2017; Janda et al, 2021). It is rich in phenylpropenoids, flavonoids, such as myricetin, naringenin, apigenin, catechin and luteolin, polysaccharides, sesquiterpenoids, triterpenoids, steroids, nitrogen components and organic acids (Bezerra et al., 2017; Gur et al., 2017; Aisa et al.,2020, Suleman et al., 2020). In addition, it has high levels of chicory acid (antiviral action), caffeic acid (Brieudes et al.,2016) quinolinic acid, succinic acid, oxalic acid and chiquimic acid (Papetti et al., 2013).

Bitter chicory leaves are used in several studies (Rani et al., 2004, Quave et al., 2008; Zhang et al., 2014; Eslami et al., 2017; Abdullah et al. 2019). In relation to the composition, total phenolic content (higher in leaves) was $865.91 \mathrm{mg}$ gallic acid equivalent/100 g (d.w.), being catechein (96.41 mg/100 g d.w.), catechol (5.13 mg/100 g d.w.), epicatachin (13.12 mg/100 g d.w.) and coumarin (11.10 mg/100 g d.w.); flavonoid contente was $112.38 \mathrm{mg}$ quercetin equivalent (QE)/100 g d.w. (Khafaf et al., 2018). Caffeic acid (1.27 mg/100 g d.w.), gallic acid (1.52 mg/100 g d.w.), 4-amino-benzoic (8.61 mg/100 g d.w.), p- 
OH-benzoic (11.93 mg/100 g d.w.), caffene (68.76 mg/100 g d.w.), ferulic acid (5.85 mg/100 g d.w.), iso-ferulic acid (49.10 mg/100 g d.w.), e-vanilic acid (131.02 mg/100 g d.w.), benzoic acid (77.50 mg/100 g d.w.), ellagic acid (91.19 mg/100 g d.w.), alpha-cumaric (7.58 mg/100 g d.w.), 3,4,5-methoxycinnamic (6.37 mg/100 g d.w.), salycilic acid (15.76 mg/100 g d.w.) and cinnamic acid $(0.70 \mathrm{mg} / 100 \mathrm{~g}$ d.w.) was identificate (Khalaf et al., 2018). Moreover, the plant is rich in chicory acid with a content of $370 \mathrm{mg} / \mathrm{kg}$ fw reported by Bahri et al. (2012). Ferioli and D’Antuono (2012) detected various sesquiterpene lactones in leaves of two different cultivars of chicory. They quantified the presence of free and bounded forms of sesquiterpene lactones, 25 and their content was: 11(S), 13-dihydrolactucin (437.4 mg/kg d.w.), lactucin (350.3 mg/kg d.w.), 8deoxylactucin (598.8 mg/kg d.w.), 11(S),13-dihydro-8-deoxylactucin (613.9 mg/kg d.w.), 11(S),13- dihydrolactucopicrin (69.5 $\mathrm{mg} / \mathrm{kg}$ d.w.) and lactucopicrin (315.7 mg/kg d.w.) (Perovic et all., 2021).

Its roots is rich in inulin, consider major natural source, it is a fructose polymer with $\beta$-(2-1)-glycosidic-linkage which is a long-chain carbohydrate. That content varies from 11-20 g inulin/100 $\mathrm{g}$ in the fresh root (Figueira et al, 2004) to $44.69 \%$ inulin on dry root weight basis (Nwagor et al, 2017). Chicory root contains higher amount of camphor, gamaterpinene and cymene $(20.74 \%, 13.24 \%$ and $15.06 \%$, respectivement). Other volatile compounds found in chicory root are octane (34.3$69.8 \%)$, nonadecane (0.3-3.9\%), aliphatic compounds and their derivates (64.1-81.3\%) and tentatively identified compounds $(4.8-22.7 \%)$ (Judzentiene \& Budiene, 2008). There are three sesquiterpene hydrocarbons, two oxygenated sesquiterpenes and nine aliphatic compounds with share of $17.1 \%, 4.8 \%$ and $78.1 \%$, respectively. According Spina et al. (2008), total phenolic contents were significantly different for wild and cultivated chicory root $(22.4$ and $35.1 \mathrm{mg}$ gallic acid equivalent $/ 100 \mathrm{~g}$, respectively). Juśkiewicz, Zduńczyk, Żary-Sikorska, Król, Milala e Jurgoński (2011) found the content of caffeoylquinic acids $(0.50 \mathrm{~g} / 100 \mathrm{~g})$, monocaffeoylquinic acids $(0.30 \mathrm{~g} / 100 \mathrm{~g})$ and Dicafeoilquinic acids $(0.20 \mathrm{~g} / 100 \mathrm{~g})$ in lyophilized chicory root. Caffeic acid $1.27 \mathrm{mg} / 100 \mathrm{~g}$ d.w. is present in chicory root with content of $35.22 \%$, protocatechuic (7.98 mg/100 g d.w.), p-hydroxybenzoic (11.04\%), iso vanillic (1.97\%, $30.66 \mathrm{mg} / 100 \mathrm{~g} \mathrm{d.w.)}$ and p-coumaric acids (9.65\%, $22.84 \mathrm{mg} / 100 \mathrm{~g}$ d.w.) were also present in chicory root (Nwafor et al., 2017). The most abundant sesquiterpene lactones isolated from the root of Cichorium intybus L. are lactucin, 8- deoxylactucin, 11(S),13-dihydro-8-deoxylactucin, lactucopicrin, 11(S),13dihydrolactucopicrin, jacquinelin, crepidiaside B, lactuside A (Willeman et al., 2014). Poli et al. (2002) mentioned that sequiterpene lactone content depends on the harvest date. This composites are target of studies with the purpose of evaluating the plant's antimicrobial activity (Quave et al., 2008).

The seeds of the species $C$. intybus were the most prevalent among the selected studies, mainly due to their rich composition demonstrated in the phytochemical analysis by other authors (Mehmood et al., 2012; Aisa et al, 2020). Tannins and flavonoids are present in Cichorium intybus L. seeds in percent of 14.53 $\pm 0.02,14.13 \%$ respectively (Abbas et al., 2012). Jurgoński, Juśbkiewicz, Zdunczyk and Bogusław (2012) determined 9.6 g phenolic compounds/100 g fresh mass including mono- and dicaffeoylquinic acids (caffeoylquinic acids (CQA), $2.8 \mathrm{~g} / 100 \mathrm{~g}$ f.w. and $6.8 \mathrm{~g} / 100 \mathrm{~g}$ f.w. respectively) and contain the smallest amount of total fructans (1.9\% d.w.) (PEROVIC et al., 2020).

The plant's stem and flowers are used on a smaller scale (Abdullah et al, 2019; Moghaddam et al., 2019). Shad et al. (2013) determined the most important compound is phenolic acids content of $2.09 \pm 0.21 \mathrm{~g} / 100 \mathrm{~g}$ d.w.

Thus, it is observed that there is a wide range of phytochemical compounds presented by C. intybus L., which are distributed in all parts of the plant. While reading the selected articles that aimed to evaluate the antimicrobial and antibiofilm activity of the species under study and other plants, it was observed that many researchers chose to use different extracting solvents, due to the different polarities of the chemical compounds, among which they ethanolics (Ahmad et al, 1998; Quave et al., 2008; Shaikh et al, 2016; Gur et al 2017), aqueous (Ahmad et al, 1998; Rani et al, 2004; Quave et al., 2008; Sharma et al, 2014; Rahman et al., 2016; Shaikh et al, 2016; Khatami et al., 2018), hexanolic (Ahamad et al, 1998; Rahman et al., 2016), methanolics (Rani et al, 2004; Mehmood et al., 2012; Abdullah et al, 2019; Moghaddam et al, 2019) ethyl acetate (Shaikh et 
al, 2016), chloroform (Rahman et al, 2016), hydroethanolic (Eslami et al., 2017) hidroethanolic (Eslami et al., 2017) and hydromethanolic (Suleman et al., 2020).

In addition, other authors extracted fractions of the prefabricated extracts, and obtained aqueous solutions (Rehman $e t$ al., 2014), ethanolic (Rehman et al, 2014), in ethyl acetate (Mehmood et al., 2012; Rahman et al., 2014), n-hexane (Mehmood et al, 2012; Rahman et al, 2014), chloroform (Mehmood et al., 2012; Rahaman et al., 2014), and n-butanol (Mehmood et al., 2012). Two studies also used low and high molecular weight fractions, which are being increasingly searched for their promising results (Spratt et al., 2012; Papetti et al., 2013).

\section{Experimental studies of the species Cichorium intybus $L$.}

Experimental studies that addressed the theme of $C$. intybus antimicrobial or antibiofilm actions are shown in Table 1.

Table 1: Characteristics Experimental Studies.

\begin{tabular}{|c|c|c|c|c|c|}
\hline AUTHOR & OBJECTS, DESIGN & MICROOGANISM & $\begin{array}{l}\text { EXTRACT/ } \\
\text { FRACTION }\end{array}$ & PART & RESULTS \\
\hline $\begin{array}{l}\text { AHMAD; } \\
\text { MEHMOOD; } \\
\text { MOHAMMAD, } \\
1998\end{array}$ & $\begin{array}{l}82 \text { Indian plant species, } \\
\text { among them the } \\
\text { Cichorium intybus, in } \\
\text { vitro }\end{array}$ & $\begin{array}{l}\text { Bacillus subtilis; } \\
\text { Escherichia coli; } \\
\text { Proteus vulgaris; } \\
\text { Salmonella typhimurium; } \\
\text { Pseudomonas aeruginosa; } \\
\text { Staphylococcus aureus }\end{array}$ & $\begin{array}{l}\text { Extracts: } \\
\text { Aqueous; } \\
\text { Ethanolic; } \\
\text { Hexanolic } \\
(20 \mathrm{~g}: 100 \mathrm{ml})\end{array}$ & Whole plant & $\begin{array}{l}\text { Ethanol extract: Action against the growth of } B \text {. } \\
\text { subtilis and } S . \text { aureus bacteria. } \\
\text { Aqueous extract: No results. } \\
\text { Hexanolic extract: No results }\end{array}$ \\
\hline $\begin{array}{l}\text { RANI; } \\
\text { KHULLAR, } \\
2004\end{array}$ & $\begin{array}{l}54 \text { plants, including } \\
\text { Cichorium intybus }\end{array}$ & $\begin{array}{c}\text { Salmonella typhi (B330) } \\
\text { Antibiotic resistent Salmonella } \\
\text { typhi } \\
\text { (MTCC 531) }\end{array}$ & $\begin{array}{l}\text { Extracts: } \\
\text { Aqueous; } \\
\text { Methanolic } \\
(50 \mathrm{mg} / \mathrm{mL})\end{array}$ & Leaf & $\begin{array}{l}\text { Methanolic extract: Moderate inhibiting activity } \\
\text { against } \text { S. typhi (B330). } \\
\text { Aqueous extract: Without inhibition. }\end{array}$ \\
\hline $\begin{array}{c}\text { QUAVE } \\
\text { ET AL., } 2008\end{array}$ & 168 plants & $\begin{array}{l}\text { Staphylococcus aureus } \\
\text { methicillin resistant }\end{array}$ & $\begin{array}{c}\text { Extract: } \\
\text { Ethanol } \\
\text { (1g: } 10 \mathrm{~mL}) \\
\text { Aqueous }(1 \mathrm{~g}: 50 \mathrm{~mL})\end{array}$ & $\begin{array}{l}\text { Basal leaves } \\
\text { Roots }\end{array}$ & $\begin{array}{l}\text { Ethanolic extract: Limited in relation to } S \text {. } \\
\text { aureus, and has shown no results in inhibiting } \\
\text { the formation of its biofilm. } \\
\text { Aqueous extract: No results. }\end{array}$ \\
\hline $\begin{array}{l}\text { MEHMOOD } \\
\text { ET AL., } 2012\end{array}$ & Cichorium intybus & $\begin{array}{l}\text { Pasteurella multocida, } \\
\text { Escherichia coli; } \\
\text { Bacillus subtilis; } \\
\text { Staphylococcus aureus; } \\
\text { Aspergillus flavus; } \\
\text { Aspergillus niger; } \\
\text { Rhizoctonia solani }\end{array}$ & $\begin{array}{c}\text { Extract: } \\
\text { Methanolic } \\
\\
\text { Fractions: } \\
\text { Ethyl acetate; } \\
\text { Chloroform; } \\
\text { N-butanol; } \\
\text { N-hexane } \\
\\
\text { (Does not report } \\
\text { concentration used) }\end{array}$ & Seed & $\begin{array}{l}\text { Methanolic extract: It showed good activity } \\
\text { against E. coli and P. multocida, but less activity } \\
\text { was shown against A. niger and B. subtilis. } \\
\text { Fraction of ethyl acetate: Strong activity against } \\
\text { E. coli. and S. aureus, but bad against A. flavus } \\
\text { and A. niger } \\
\text { Chloroform fraction: Good against S. aureus } \\
\text { and E. coli, but bad against B. subtilis and P. } \\
\text { multocida. } \\
\text { N-butanol fraction: Good against } P \text {. multocida } \\
\text { and S. aureus, but bad against A. flavus and A. } \\
\text { niger. } \\
\text { N-hexane fraction: Good against S. aureus and } \\
\text { E. coli, but bad against A. flavus and B. subtilis. } \\
\text { Satisfactory action of Low Molecular Mass: }\end{array}$ \\
\hline ET AL., 2012 & $\begin{array}{c}\text { (Camellia sinensis) } \\
\text { Cranberry juice } \\
\text { (Vaccinium } \\
\text { macrocarpo) } \\
\text { Rasberry } \\
\text { (Rubus idaeus) } \\
\text { Shiitake mushrooms } \\
\text { (Lentimula edodes) } \\
\text { Red chicory } \\
\text { (Cichorium intybus) } \\
\text { Beer } \\
\text { (Guinness) }\end{array}$ & $\begin{array}{l}\text { Actinomyces naeslundii; } \\
\text { Fusobacterium nucleatum; } \\
\text { Prevotella intermedia; } \\
\text { Streptococcus mutans; } \\
\text { Lactobacillus casei; } \\
\text { Veillonella dispar; } \\
\text { Neisseria subflava }\end{array}$ & $\begin{array}{l}\text { Low molecular mass } \\
\text { High molecular mass } \\
\text { (Does not report } \\
\text { concentration or } \\
\text { solvent used) }\end{array}$ & & $\begin{array}{l}\text { Preventing the formation of biofilm of species } \\
\text { related to gingivitis, disrupting pre-existing } \\
\text { biofilms of dental caries bacteria and inhibiting } \\
\text { the adhesion and invasion of the bacterium A. } \\
\text { naeslundii to gingival epithelial cells. }\end{array}$ \\
\hline
\end{tabular}


Research, Society and Development, v. 11, n. 2, e10911225384, 2022

(CC BY 4.0) | ISSN 2525-3409 | DOI: http://dx.doi.org/10.33448/rsd-v11i2.25384

\begin{tabular}{|c|c|c|c|c|c|}
\hline \multirow[b]{2}{*}{$\begin{array}{c}\text { PAPETTI } \\
\text { ET AL., } 2013\end{array}$} & \multirow[b]{2}{*}{$\begin{array}{l}\text { Quinolinic acid } \\
\text { Succinic acid } \\
\text { Oxalic acid } \\
\text { Shikimic acid }(C \text {. } \\
\text { intybus compounds) }\end{array}$} & \multirow[b]{2}{*}{$\begin{array}{c}\text { Actinomyces naeslundii; } \\
\text { Prevotella intermedia; } \\
\text { Streptococcus mutans; } \\
\text { Fusobacterium nucleatum; } \\
\text { Veilonella dispar; } \\
\text { Neisseria subflava; } \\
\text { Streptococcus sanguinis; } \\
\text { Lactobacillus casei }\end{array}$} & \multirow[b]{2}{*}{$\begin{array}{l}\text { Fraction: } \\
\text { Low molecular mass } \\
\text { (Does not report } \\
\text { concentration or } \\
\text { solvent used) }\end{array}$} & & \multirow[b]{2}{*}{$\begin{array}{l}\text { Succinic acid: inhibited biofilm formation of all } \\
\text { bacteria } \\
\text { Quinolinic acid: Inhibited the biofilm formation } \\
\text { of microorganisms, especially S. mutans and P. } \\
\text { intermedia. } \\
\text { Shikimic acid: It broke the biofilm of S. mutans } \\
\text { and A. naeslundii. }\end{array}$} \\
\hline & & & & & \\
\hline $\begin{array}{l}\text { SHARMA } \\
\text { ET AL., } 2014\end{array}$ & $\begin{array}{l}\text { Cichorium intybus } \\
\text { Coffe }\end{array}$ & Streptococcus mutans & $\begin{array}{l}\text { Extract: } \\
\text { Aqueous } \\
(20 \mathrm{~g}: 250 \mathrm{~mL})\end{array}$ & - & $\begin{array}{l}\text { C. intybus pure:It showed a smaller number of } \\
\text { bacterial colonies than the other groups. }\end{array}$ \\
\hline $\begin{array}{c}\text { ZHANG } \\
\text { ET AL., } 2014\end{array}$ & $\begin{array}{l}\text { Chicory acid (one of } \\
\text { the main compounds } \\
\text { in C. intybus) }\end{array}$ & Hepatitis B virus & $\begin{array}{l}\text { (Does not report the } \\
\text { solvent used) }\end{array}$ & Sheets & Chicory acid: Effective against the virus. \\
\hline \multirow[t]{2}{*}{$\begin{array}{l}\text { REHMAN } \\
\text { ET AL., } 2014\end{array}$} & Cichorium intybus & $\begin{array}{l}\text { Escherichia coli; } \\
\text { Klebsiella pneumoniae; } \\
\text { Pseudomonas aeruginosa; } \\
\text { Staphylococcus epidermidis; } \\
\text { Staphylococcus aureus } \\
\text { (resistente à meticilina); }\end{array}$ & $\begin{array}{c}\text { Extract: } \\
\text { Ethanol }(3 \mathrm{mg} / \mathrm{mL}) \\
\\
\text { Fractions: } \\
\text { N-hexane; } \\
\text { Chloroform; }\end{array}$ & Whole plant & $\begin{array}{l}\text { Ethanol extract: Activity against } P \text {. aeruginosa, } \\
\text { A. niger, A.flavus and } S \text {. aureus. } \\
\text { Fractions of n-hexane and chloroform: Activity } \\
\text { in } P \text {. aeruginosa, } K \text {. pneumoniae, } B \text {. subtilis, } S \text {. } \\
\text { epidermidis and } S \text {. aureus. } \\
\text { Aqueous fraction: Activity against } P \text {. }\end{array}$ \\
\hline & & $\begin{array}{l}\text { Bacillus subtilis; } \\
\text { Aspergillus flavus; } \\
\text { Aspergillus niger; } \\
\text { Aspergillus fumigatus; } \\
\text { Fusarium solani }\end{array}$ & $\begin{array}{l}\text { Watery; } \\
\text { Ethyl acetate } \\
(4 \mathrm{mg} / \mathrm{mL})\end{array}$ & & $\begin{array}{l}\text { aeruginosa, A. niger, A. fumigatus, A. flavus and } \\
\text { S. aureus. } \\
\text { Fraction of ethyl acetate: Activity against } F \text {. } \\
\text { solani, B. subtilis and S. aureus. }\end{array}$ \\
\hline $\begin{array}{l}\text { RAHMAN } \\
\text { ET AL., } 2016\end{array}$ & Cichorium intybus & $\begin{array}{l}\text { Pseudomonas aeruginosa; } \\
\text { Staphylococcus aureus; } \\
\text { Escherichia coli; } \\
\text { Acinetobacter baumannii }\end{array}$ & $\begin{array}{c}\text { Extracts: } \\
\text { Aqueous; } \\
\text { Chloroform; } \\
\text { Ethanolic; } \\
\text { Hexanolic } \\
(25 \mathrm{~g} / \mathrm{L} \text { in DMSO })\end{array}$ & Seed & $\begin{array}{l}\text { Aqueous extract: Very satisfactory results on } P \text {. } \\
\text { aeruginosa and A. baumannii. } \\
\text { Chloroform, ethanolic and hexanolic extracts: } \\
\text { Good results in inhibiting the bacteria S. aureus. }\end{array}$ \\
\hline $\begin{array}{l}\text { SHAIKH; RUB; } \\
\text { SASIKUMAR, } \\
\quad 2016\end{array}$ & Cichorium intybus & $\begin{array}{l}\text { Staphylococcus aureus; } \\
\text { Pseudomonas aeruginosa; } \\
\text { Candida albicans; } \\
\text { Escherichia coli }\end{array}$ & $\begin{array}{l}\text { Extracts: } \\
\text { Ethyl acetate; } \\
\text { Aqueous; } \\
\text { Ethanol } \\
(1 \mathrm{mg} / \mathrm{mL})\end{array}$ & Seed & $\begin{array}{l}\text { Ethyl acetate extract: Inhibited the growth of } P \text {. } \\
\text { aeruginosa and } S \text {. aureus. } \\
\text { Ethanolic extract: affected the fungus } C \text {. } \\
\text { albicans and the bacterium E. coli. } \\
\text { Aqueous extract: Maximum activity on } S \text {. aureus. }\end{array}$ \\
\hline $\begin{array}{c}\text { GUR } \\
\text { ET AL., } 2017\end{array}$ & $\begin{array}{l}\text { Cannabis sativa } \\
\text { Cichorium intybus } \\
\text { Lavandula stoechas } \\
\text { Valeriana officinalis } \\
\text { Glycyrrhiza glabra }\end{array}$ & $\begin{array}{c}\text { Bacillus subtilis; } \\
\text { Candida albicans; } \\
\text { Enterobacter aerogenes; } \\
\text { Enterococcus faecalis; } \\
\text { Enterococcus faecium; } \\
\text { Escherichia coli; } \\
\text { Klebsiella pneumoniae; } \\
\text { Pseudomonas aeruginosa; } \\
\text { Pseudomonas fluorescens; } \\
\text { Salmonella enteritidis; } \\
\text { Salmonella infantis; } \\
\text { Salmonella kentucky; } \\
\text { Salmonella typhimurium; } \\
\text { Staphylococcus aureus; } \\
\text { Staphylococcus epidermidis }\end{array}$ & $\begin{array}{c}\text { Extract: } \\
\text { Ethanol } \\
(10-30 \mathrm{~g} / 250 \mathrm{~mL})\end{array}$ & Whole plant & $\begin{array}{l}\text { Ethanol extract: Action against C. albicans and } \\
\text { E. faecalis. } \\
\text { No results: E. aerogenes, E. faecium, E. coli, K. } \\
\text { pneumonia, P. aeruginosa, P. fluorescence, S. } \\
\text { infantil, S. kentucky, S. aureus, S. epidermidis }\end{array}$ \\
\hline $\begin{array}{l}\text { ESLAMI } \\
\text { ET AL., } 2017\end{array}$ & Cichorium intybus & $\begin{array}{l}\text { Candida glabrata; } \\
\text { Candida krusei }\end{array}$ & $\begin{array}{l}\text { Extract: } \\
\text { Hydroethanolic } \\
\text { (1g: } 100 \mathrm{~mL})\end{array}$ & Leaf & $\begin{array}{l}\text { Extract: Greater sensitivity of the C. krusei } \\
\text { fungus, however a low Minimum Inhibitory } \\
\text { Concentration was observed for the two fungi. }\end{array}$ \\
\hline $\begin{array}{c}\text { ZHU } \\
\text { ET AL., } 2018\end{array}$ & Chicory acid & $\begin{array}{l}\text { Lactobacillus plantarum; } \\
\text { rhamnosus; } \\
\text { crispatus; } \\
\text { Bifidobacterium longum; } \\
\text { Salmonella enteritidis; } \\
\text { Shigella typhimurium; } \\
\text { Escherichia coli; } \\
\text { Listeria monocytogenes }\end{array}$ & $\begin{array}{l}\text { (Does not report } \\
\text { concentration or } \\
\text { solvent used) }\end{array}$ & - & $\begin{array}{l}\text { Chicory acid: did not affect the growth of } L \text {. } \\
\text { plantarum, } L \text {. rhamnosus, } L \text {. crispatus and } B \text {. } \\
\text { longum. } \\
\text { The concentrations of } 120 \text { and } 160 \mathrm{mM} \text { of the } \\
\text { acid inhibited the growth of the pathogens } S \text {. } \\
\text { enteritidis, } S \text {. typhimurium, E. coli and } L \text {. } \\
\text { monocytogenes. }\end{array}$ \\
\hline $\begin{array}{l}\text { KHATAMI } \\
\text { ET AL., } 2018\end{array}$ & $\begin{array}{l}\text { Cichorium intybus } \\
\text { (Silver nanospheres } \\
\text { biosynthesized with } \\
\text { the plant) }\end{array}$ & $\begin{array}{l}\text { Pseudomonas aeruginosa; } \\
\text { Klebsiella pneumoniae; } \\
\text { Acinetobacter baumannii; } \\
\quad \text { Fusarium solani }\end{array}$ & $\begin{array}{l}\text { Extract: } \\
\text { Aqueous } \\
(7 \mathrm{~g}: 100 \mathrm{~mL})\end{array}$ & Seed & $\begin{array}{l}\text { Nanospheres: Excellent antibacterial activity } \\
\text { against selected bacteria, with low values of } \\
\text { MIC, MBI, highlighting the results against the } \\
\text { fungus F. solani. }\end{array}$ \\
\hline $\begin{array}{l}\text { ABDULLAH; } \\
\text { AL-SAEDI; } \\
\text { SALMAN, } 2019\end{array}$ & Cichorium intybus & $\begin{array}{l}\text { Staphylococcus aureus; } \\
\text { Pseudomonas aeruginosa; } \\
\text { Escherichia coli; } \\
\text { Klebsiella spp.; } \\
\text { Proteus vulgaris; } \\
\text { Enterobacter spp; } \\
\text { Lactobacillus spp; } \\
\text { Streptococcus faecalis }\end{array}$ & $\begin{array}{l}\text { Extract: } \\
\text { Methanolic } \\
(30 \mathrm{~g}: 300 \mathrm{~mL})\end{array}$ & $\begin{array}{l}\text { Leaf } \\
\text { Stalk } \\
\text { Seed }\end{array}$ & $\begin{array}{l}\text { Best results: Concentration of } 1000 \mathrm{mg} / \mathrm{mL} \text { of } \\
\text { methanolic extract from the leaves against } S \text {. } \\
\text { aureus, } P \text {. aeruginosa, Klebsiella spp., } P . \\
\text { vulgaris, Lactobacillus spp. and } S \text {. faecalis. } \\
\text { Extracts produced from the stem and seeds did } \\
\text { not produce satisfactory results. }\end{array}$ \\
\hline $\begin{array}{l}\text { MOGHADDAM } \\
\text { ET AL., } 2019\end{array}$ & $\begin{array}{l}\text { Fifteen species of } \\
\text { medicinal herbs, } \\
\text { including Cichorium } \\
\text { intybus }\end{array}$ & $\begin{array}{l}\text { Bacillus subtilis; } \\
\text { Staphylococcus aureus; } \\
\text { Pseudomonas aeruginosa; } \\
\text { Escherichia coli; } \\
\text { Candida albicans }\end{array}$ & $\begin{array}{l}\text { Extract: } \\
\text { Methanolic } \\
(2 \mathrm{~g}: 20 \mathrm{~mL})\end{array}$ & Flowers & $\begin{array}{l}\text { C. intybus: Antibacterial activity against } B \text {. } \\
\text { subtilis, E. coli and } S \text {. aureus, but there was no } \\
\text { apparent action against } P \text {. aeruginosa and } C \text {. } \\
\text { albicans }\end{array}$ \\
\hline $\begin{array}{l}\text { SULEIMAN, } \\
2020\end{array}$ & $\begin{array}{l}\text { Cichorium intybus } \\
\text { Cinnamomum } \\
\text { camphora } \\
\text { Commiphora myrrha } \\
\text { Foeniculum vulgare }\end{array}$ & $\begin{array}{l}\text { Candida albicans; lipolytica; } \\
\text { Enterococcus faecalis; } \\
\text { Streptococcus mutans; } \\
\text { Micrococcus sp.; } \\
\text { Enterobacter cloaca; }\end{array}$ & $\begin{array}{l}\text { Extract: } \\
\text { Hydromethanolic } \\
\text { (10g: } 100 \mathrm{~mL})\end{array}$ & Seed & C. intybus: Higher results in Candida lipolytica. \\
\hline
\end{tabular}


In vitro studies were carried out in order to evaluate the effectiveness of Cichorium intybus extracts on the bacterium Staphylococcus aureus. As noted, this microorganism is predisposed to offer resistance to antibiotics such as methicillin (MRSA), in addition to being a major cause of nosocomial pneumonia and bacterial endocarditis. Many studies have found that the plant acts with the extracts of ethyl acetate, chloroform, n-butanol and n-hexane (Mehmood et al., 2012; Rahman et al., 2014, Rehmen et al., 2016), ethanolic and hexanolic (Ahman et al., 1998; Rehman et al., 2014), aqueous (Rahman et al., 2016), and methanolic (Abdullah et al., 2019; Moghaddam et al, 2019). Quave et al. (2008), when using ethanolic extracts from basal leaves and roots, concluded that the bacteriostatic activity of bitter chicory was limited in relation to MRSA, and did not show results in inhibiting the formation of its biofilm.

Ahmad et al. (1998) found inhibition of the growth of Bacillus subtilis from the ethanolic extract, a bacterium found in dental biofilm. The results of this study differ from Rehman et al. (2014) and Gur et al. (2017) who did not observe the plant's effect on the microorganism in this extract. However, Rehman et al. (2014) observed activity in the fractions of nhexane, chloroform and ethyl acetate on B. subtilis. In methanolic extract, Mehmood et al. (2012) did not observe antibacterial activity on B. subtilis, a result different from that found by Moghaddam et al. (2019). The findings can be explained by the different parts of the plant that were used in each study, as well as the concentrations of the extract that were used in the in vitro studies.

In the case of the genus Salmonella spp., Which is related to intestinal infections, Rani et al. (2004) found action in the methanolic extract, made from the leaves of C. intybus, on the serovariety Salmonella typhi. Zhu et al. (2018), studying the action of chicoric acid, one of the most abundant organic acids in bitter chicory, also found promising results on a bacterium of the genus, S. enteritidis, in concentrations of 120 and $160 \mathrm{mM}$ of the acid. Such antimicrobial action was not observed in the study by Ahmad et al. (1998). Gur et al. (2017) did not identify in the aqueous extracts, ethyl acetate and ethanolic, produced from the seeds of the plant, action on the microorganisms S. enteritidis, S. infantil, S. kentucky and S. typhimurium. Suleiman (2020), using the hydromethanolic extract of seeds from the plant on S. typhimurium also did not find this effect. This can be explained by the different parts of $C$. intybus used in the study, concentration of extracts and serotypes researched.

The microorganism Escherichia coli, involved in infections of sterile tissues (such as the urinary tract) was used to evaluate the antimicrobial activity of Cichorium intybus. Mehmood and collaborators (2012), in their study with the seed of the plant, obtained promising results on the bacterium in fractions of ethyl acetate, chloroform and methanolic extract, as well as Moghaddam et al. (2019), who observed in the same extract inhibitory effect of the bacteria. Shaikh et al. (2016) found the action of ethanolic extract on E. coli. Other authors have not identified such an action (Ahamam et al., 1998; Rehman et al., 2014; Rahman et al., 2016; Gur et al., 2017; Abdallah et al, 2019), which can be justified by the use of different parts of the plant, concentrations and solvents.

With regard to the microorganism Pseudomonas aeruginosa, frequently associated with pneumonia in people with mechanical ventilation, most of the selected studies found the action of at least one extract or fraction on the bacterium, the most relevant being the fractions of n-hexane and chloroform (Rehman et al., 2014), aqueous extracts (Rahman et al., 2016), ethyl acetate (Shaikh et al., 2016) and methanolic (Abdullah et al.,2019). Khatami et al. (2018) demonstrated the effectiveness of nanospheres synthesized from the seeds of bitter chicory on the bacteria in their in vitro study. Ahmad et al. (1998), Gur et al. (2017) and Moghaddam et al. (2019) did not find the same results, and explain that further studies on the use of the ethanolic and methanolic extracts of Cichorium intybus on P. aeruginosa are still needed.

The main bacterium involved in the development of caries disease, Streptococcus mutans, was investigated in an in vitro study by Spratt et al. (2012), who sought to elucidate the antibacterial activity of bitter chicory on the microorganism in question. Its results with low molecular weight fractions were satisfactory, with the disruption of pre-existing biofilms from the bacteria S. mutans and L. casei (also involved in tooth decay). Papeti et al. (2013) also found action in the low molecular 
weight fraction of succinic, quinolinic and chiquimic inhibition of the S. mutans biofilm, while Sharma et al. (2014), when comparing the effects of bitter chicory with coffee, found the same results in inhibiting the bacteria. Suleiman (2020), in his study using the hydromethanolic extract of the plant's seeds, did not observe this same action on $S$. mutans. It is observed that the action of low molecular weight fractions demonstrated promising effects on S. mutans.

Still related to oral diseases, two studies aimed to elucidate the action of low molecular weight fractions of Cichorium intybus on the microorganisms Streptococcus sanguinis, Actinomyces naeslundii, Fusobacterium nucleatum and Prevotella intermedia, involved with gingivitis. Spratt et al. (2012) observed that the fractions prevented the formation of the biofilm of the species, in addition to inhibiting the adhesion and invasion of the bacterium A. naeslundii to the gingival epithelial cells. The results of Papeti et al. (2013) with the organic acids present in the plant (quinolinic, succinic, oxalic and chiquimic acids) entered into consensus with Spratt et al. (2012), as they pointed to the effectiveness of succinic and quinolinic acids in inhibiting all bacteria, with emphasis on $P$. intermedia in quinolinic acid. In addition, the shikimic acid presented a rupture of the biofilm of $A$. naeslundii, thus showing the effectiveness of the plant on the growth and biofilm of the microorganisms present in gingivitis.

When understanding that biofilm constitutes a complex microbial community and dysbiosis causes oral problems as well as in face of the unviability of the use of antimicrobials in a continuous way, due to the side effects caused as the dental staining due to the use of chlorhexidine continuously; the search for alternatives becomes inevitable (Marsh \& Martin, 2018). In terms of the plant's antiviral activity, Zhang et al. (2014) studied one of the main compounds of the C. intybus plant, chicory acid, seeking to evaluate its effects on the Hepatitis B virus. For the preparation of the extract, its leaves were used. After the analysis, it was observed that the acid was effective against the virus, so it could be improved and later used as an antiviral agent. Brieudes et al. (2016) also highlighted the antiviral action of this organic acid due to its action against the HIV virus.

Regarding the antifungal activity of $C$. intybus, most of the results observed in the studies demonstrate a good action of the aqueous, ethanolic and ethyl acetate fractions against Aspergillus flavus, Aspergillus niger, Aspergillus fumigatus and Fusarium solani, (Rehman et al., 2014; Khatami et al., 2018). Ethil acetate fraction inhibiting the total growth of the first three species presented and aqueous to F.solani (Rehman et al., 2014), the mechanism of action of these fractions has not yet been explored by the studies but it is essential to find alternatives since the antifungal resistance rises remarkably, requiring alternatives (Buzina et al., 2013).

Mehmood and collaborators (2012), however, found no action of methanolic extract or fractions of ethyl acetate, chloroform, n-butanol and n-hexane on the fungi Aspergillus flavus, Aspergillus niger and Rhizoctonia solani. Regarding the fungi Candida albicans, C. krusei and C. lipolytica, the ethanolic, hydroethanolic, methanolic and hydromethanolic extracts were effective (Shaikh et al., 2016; Gur et al., 2017; Eslami et al., 2017; Moghaddam et al., 2019; Suleman et al., 2020).

On the toxicity of the plant, Spratt et al. (2012) found no cytotoxic activity of bitter chicory tested by the trypan blue exclusion test, in agreement with Eslami et al. (2017), who cited the low toxicity reported in laboratory studies performed on rats. Furthermore, Zhang et al. (2014) did not observe cytotoxic activity of chicory acid, one of the main compounds of the plant, on hepatocytes, agreeing with previous authors.

In view of the observed results, it is possible to notice a wide antimicrobial and antibiofilm action of $C$. intybus in the most polar solvents, such as ethanol and methanol, intermediate polarity, such as ethyl acetate and chloroform, and low molecular weight fractions. The microorganisms that were most sensitive to the action of the plant and its chemical components were the bacteria S. aureus, S. mutans, P. aeruginosa, B. subtilis, S. sanguinis, A. naeslundii, F. nucleatum and P. intermedia. 
The fungi most affected were $C$. albicans, $C$. lipolytica, $C$. krusei and $F$. solani. In addition, an antibiofilm action was observed, mainly in low molecular weight fractions, in bacteria S. mutans, L. casei, S. nguinis, A. naeslundii, F. nucleatum and $P$. intermedia. Finally, a study also revealed a good action of chicory acid on the Hepatitis B virus.

However, there is no consensus in the literature on the concentration of extracts, the part of the plant and the solvents to be used. Thus, it is necessary to standardize the methodology of the studies to better compare the results, in addition to the selection of microorganisms more sensitive to the bioactive compounds of the species so that promising results are found that prove the antimicrobial and antibiofilm action of Cichorium intybus.

\section{Conclusion}

In view of the observed results, it is possible to notice a wide antimicrobial and antibiofilm action of C. intybus in the most polar solvents, such as ethanol and methanol, intermediate polarity, such as ethyl acetate and chloroform, and low molecular weight fractions. The microorganisms that were most sensitive to the action of the plant and its chemical components were the bacteria $S$. aureus, S. mutans, P. aeruginosa, B. subtilis, S. sanguinis, A. naeslundii, F. nucleatum and $P$. intermedia. The fungi most affected were $C$. albicans, C. lipolytica, $C$. krusei and F. solani. In addition, an antibiofilm action was observed, mainly in low molecular weight fractions, in bacteria S. mutans, L. casei, S. sanguinis, A. naeslundii, F. nucleatum and P. intermedia. Finally, a study also revealed a good action of chicory acid on the Hepatitis B virus.

However, there is no consensus in the literature on the concentration of extracts, the part of the plant and the solvents to be used. Thus, it is necessary to standardize the methodology of the studies to better compare the results, in addition to the selection of microorganisms more sensitive to the bioactive compounds of the species so that promising results are found that prove the antimicrobial and antibiofilm action of Cichorium intybus $\mathrm{L}$.

\section{References}

Abdullah, B. H., Al-Saedi, F. \& Salman, A. E. (2019). Effects of Cichorium intybus Methanolic extracts on Some Clinical Bacterial Isolates. Indian Journal of Public Health Research \& Development. 10(2), 829-33.

Ahmad, I., Mehmood, Z. \& Mohammad, F. (1998). Screening of some Indian medicinal plants for their antimicrobial properties. Journal of ethnopharmacology. 62(2)183-193.

Aisa, H.A., Xin, X. \& Tang, D. (2020). Chemical constituents and their pharmacological activities of plants from Cichorium genus. Chinese Herbal Medicines.12(3),224-236.

Babaei, H. et al (2018). Effects of chicory leaf extract on serum oxidative stress markers, lipid profile and periodontal status in patients with chronic periodontitis. Journal of the American College of Nutrition. 37(6)479-486.

Bahmani, M. et al. (2015) Chicory: A review on ethnobotanical effects of Cichorium intybus L. Journal of Chemical and Pharmaceutical Sciences. 8(4)672682 .

Bezerra, A. S. et al. (2017). Composição nutricional e atividade antioxidante de plantas alimentícias não convencionais da região Sul do Brasil. Arquivos Brasileiros de Alimentação. 1(1),182-8.

Brieudes, V. et al. (2016). Phytochemical analysis and antioxidant potential of the phytonutrient-rich decoction of Cichorium spinosum and C. intybus. Planta medica. 82(11) 1070-1078.

Canesi, L. et al. (2011). Effects of fruit and vegetable low molecular mass fractions on gene expression in gingival cells challenged with Prevotella intermedia and Actinomyces naeslundii. Journal of Biomedicine and Biotechnology.2011(5),1-8.

Chamani, G. et al. (2011). Evaluation of effects of Zingiber officinale on salivation in rats. Acta Medica Iranica; 49(6),336-340.

Eldin, S. \& Dunford, A. Fitoterapia: na atenção primária à saúde. Editora Manole Ltda, 2001.

Eslami, H. et al. (2017) Evaluation of the Antifungal Effect of Chicory Extracts on Candida Glabrata and Candida Krusei in a Laboratory Environment. The Journal of Contemporary Dental Practice. 18(11), 1014-1020.

Francisco, K. S. F. (2010) Fitoterapia: Uma opção para o tratamento odontológico. Revista Saúde. 4(1), 18-24.

Gazzani, G, Daglia, M, \& Papetti. (2012) A. Food components with anticaries activity. Current Opinion in Biotechnology. 23(2),153-159. 
Gur, M. et al. (2017). Determination of Some Flavonoids and Antimicrobial Behaviour of Some Plants' Extracts. Indian Journal of Pharmaceutical Education and Research. 51(3), S225-S229.

Khatami, M. et al. (2018) Antifungal and antibacterial activity of densely dispersed silver nanospheres with homogeneity size which synthesized using chicory: an in vitro study. Journal de Mycologie Medicale. 18(4)637-644.

Mehmood, N. et al. (2012) Antioxidant, antimicrobial and phytochemical analysis of cichoriumintybus seeds extract and various organic fractions. Iranian Journal of Pharmaceutical Research: IJPR. 11(4), 1145-1149.

Moghaddam, N. S. et al. (2019) Antimicrobial screening of some selected Turkish medicinal plants. Pakistan Journal of Pharmaceutical Sciences. 32(2), 947951 .

Papetti, A. et al. (2013) Identification of organic acids in Cichorium intybus inhibiting virulence-related properties of oral pathogenic bacteria. Food Chemistry $138(2-3)$ 1706-1712.

Quave, C L., Plano, L. R.., Pantuso, T., \& Bennett B. C. (2008) Effects of extracts from Italian medicinal plants on planktonic growth, biofilm formation and adherence of methicillin-resistant Staphylococcus aureus. J Ethnopharmacol.Aug 13;118(3):418-28.

Rahman, H. et al. (2016) Ethnomedicinal Cichorium intybus seed extracts: An impending preparation against multidrug resistant bacterial pathogens. Jundishapur. Journal of Microbiology. 9(11)., 1-10.

Rani, P, Khullar, N. (2004) Antimicrobial evaluation of some medicinal plants for their anti-enteric potential against multi-drug resistant Salmonella typhi. Phytotherapy Research: An International Journal Devoted to Pharmacological and Toxicological Evaluation of Natural Product Derivatives. 18(8), 670-673.

Rehmanm A. et al. (2014) Antibacterial and antifungal study of Cichorium intybus. Asian Pacific Journal of Tropical Disease. 4(1) S943-S945.

Saedd, M. et al. (2017). Chicory (cichorium intybus) herb: Chemical composition, pharmacology, nutritional and healthical applications. International Journal of Pharmacology. 13(4), 351-360.

Shaikh, T., Rub, R. A, \& Sasikumar, S. (2016). Antimicrobial screening of Cichorium intybus seed extracts. Arabian Journal of Chemistry. 9(1), S1569S1573.

Sharma, R. et al. (2014) Antimicrobial and anti-adherence activity of various combinations of coffee-chicory solutions on Streptococcus mutans: An in-vitro study. Journal of Oral and Maxillofacial Pathology: JOMFP. 18(2).201-204.

Spratt, DA. et al. (2012). Evaluation of plant and fungal extracts for their potential antigingivitis and anticaries activity. BioMed Research International, v. $2012,2012$.

Street, R. A., Sidana, J. \& Prinsloo, G. (2013). Cichorium intybus: Traditional uses, phytochemistry, pharmacology, and toxicology. Evidence-Based Complementary and Alternative Medicine.

Suleiman, W. B. (2020). In vitro estimation of superfluid critical extracts of some plants for their antimicrobial potential, phytochemistry, and GC-MS analyses. Annals of Clinical Microbiology and Antimicrobials. 19(1), 1-12.

Zhang, H. et al. (2014). Evaluation of hepatocyteprotective and anti-hepatitis B virus properties of Cichoric acid from Cichorium intybus leaves in cell culture. Biological and Pharmaceutical Bulletin. b14-00137.

Zhu, X. et al. (2018) Evaluation of the potential of chicoric acid as a natural food antioxidant. Experimental and therapeutic medicine. $16(4), 3651-3657$. 